\title{
PROTOCOLO PARA CONTROLE DE EFICÁCIA TERAPÊUTICA EM DISFAGIA OROFARÍNGEA NEUROGÊNICA (PROCEDON)
}

\author{
Efficacy control protocol in oropharyngeal dysphagia
}

\author{
Roberta Gonçalves da Silva ${ }^{(1)}$, Adriana Gomes Jorge ${ }^{(2)}$, Fernanda Matias Peres ${ }^{(3)}$, \\ Paula Cristina Cola ${ }^{(4)}$, Ana Rita Gatto ${ }^{(5)}$, André Augusto Spadotto ${ }^{(6)}$
}

\begin{abstract}
RESUMO
Objetivo: apresentar uma proposta para o controle de eficácia terapêutica em disfagia orofaríngea neurogênica. Métodos: o protocolo foi proposto em concordância com a literatura atual e aplicado em um indivíduo pós-acidente vascular encefálico (AVE) isquêmico à direita, comprovado por tomografia computadorizada, com disfagia orofaríngea grave crônica, gênero masculino, 66 anos, apresentando aspiração laringotraqueal e em uso de sonda nasoentérica exclusiva pré-fonoterapia. Para controle da eficácia terapêutica do programa de reabilitação fonoaudiológica foi aplicado, pré e pós-fonoterapia, a classificação do grau de comprometimento da disfagia orofaríngea, Functional Oral Intake Scale (FOIS), a avaliação videofluoroscópica da deglutição com medida do tempo de trânsito faríngeo (TTF) da deglutição por meio de software e da percepção do indivíduo. Resultados: na pré-fonoterapia verificou-se disfagia orofaríngea grave, FOIS nível 1, presença de aspiração laringotraqueal para mais de uma consistência e tempo de trânsito faríngeo de 13 segundos. Após fonoterapia verificouse disfagia orofaríngea moderada, FOIS nível 5, ausência de aspiração laringotraqueal e TTF de 4 segundos. Conclusão: o protocolo proposto foi capaz de avaliar a eficácia da reabilitação na disfagia orofaríngea neurogênica neste indivíduo pós-acidente vascular encefálico, tanto para mensurar as mudanças ocorridas na fisiopatologia da deglutição quanto na ingestão oral e na percepção do indivíduo. Outros estudos com populações distintas são necessários, sendo que novas propostas devem ainda refletir a inclusão da condição nutricional e pulmonar do indivíduo no controle de eficácia em disfagia orofaríngea.
\end{abstract}

DESCRITORES: Transtornos de Deglutição; Reabilitação; Eficácia

(1) Fonoaudióloga; Professora Doutora do Departamento de Fonoaudiologia da Universidade Estadual Paulista, UNESP, Marília, SP, Brasil; Doutora em Fisiopatologia em Clínica Médica pela Faculdade de Medicina de Botucatu da Universidade Estadual Paulista.

(2) Fonoaudióloga do Hospital Estadual de Bauru, SP, Brasil.

(3) Fonoaudióloga do Hospital Estadual de Bauru, SP, Brasil.

(4) Fonoaudióloga; Doutoranda do Departamento de Cirurgia da Faculdade de Medicina de Botucatu da Universidade Estadual Paulista, UNESP, Botucatu, SP, Brasil.

(5) Fonoaudióloga do Departamento de Neurologia e Neuropsiquiatria da Faculdade de Medicina de Botucatu da Universidade Estadual Paulista, UNESP, Botucatu, SP, Brasil; Mestranda do Departamento de Cirurgia da Faculdade de Medicina de Botucatu da Universidade Estadual Paulista.

(6) Bacharel em Sistemas da Informação; Mestre em Bioengenharia pela Universidade de São Paulo; Doutor em Engenharia Elétrica pela Universidade de São Paulo.

Conflito de interesses: inexistente

\section{INTRODUÇÃO}

A eficácia da reabilitação fonoaudiológica na disfagia orofaríngea, questionada desde a década de 70 , é uma das áreas de investigação científica que têm recebido pouco investimento por parte dos pesquisadores ao longo do desenvolvimento das pesquisas nesta temática.

Inicialmente é possível compreender esta questão ao considerar que a pesquisa que envolve a reabilitação possui inúmeras variáveis, tais como o tipo de doença, o topodiagnóstico da lesão, a faixa etária, a escolaridade, a presença de questões cognitivas associadas ou não a prejuízos motores, tempo do ictus, fase de recuperação espontânea e as questões éticas com grupo controle e muitas outras. Enfim, a pesquisa em reabilitação necessita 
ser construída com uma metodologia que exige rigorosos critérios para controle de variáveis, tornando inicialmente a realização de estudos de casos uma forma coerente para atingir tal propósito ${ }^{1,2}$.

No final da década de 80 os critérios de eficácia utilizados na reabilitação da disfagia ainda não haviam sido satisfatoriamente delineados, sendo que as dificuldades de pesquisa nesta área também estavam relacionadas à ausência de consenso sobre os critérios que deveriam mensurar a eficácia da reabilitação na disfagia orofaríngea ${ }^{3-5}$.

Dentre esses critérios para mensurar a eficácia da reabilitação em disfagia orofaríngea foram descritos procedimentos que auxiliariam este controle, como a avaliação objetiva da deglutição, escalas de grau de comprometimento da disfagia orofaríngea ${ }^{6-12}$ e mais recentemente escalas funcionais de controle de ingestão oral ${ }^{13} \mathrm{e}$ o uso de software para medir tempo de trânsito orofaríngeo ${ }^{14,15}$, inclusive pós-fonoterapia ${ }^{16}$.

As doenças cerebrovasculares são consideradas a primeira causa de óbito no mundo e a segunda no Brasil, sendo responsável por inúmeras sequelas que produzem alto grau de incapacidade e dentre elas a disfagia orofaríngea. A incidência de disfagia orofaríngea nas doenças cerebrovasculares é descrita com uma variação de $40 \%$ a 90\% ${ }^{17-20}$.

A eficácia da reabilitação na disfagia orofaríngea após-acidente vascular encefálico (AVE) necessita de estudos randomizados para que as conclusões identifiquem para quais pacientes e com qual tratamento a reabilitação fonoaudiológica para disfagia orofaríngea em AVE é eficaz ${ }^{21-23}$.

Os critérios para controle da eficácia da reabilitação vêm sendo discutidos desde a década de 80 e concluiu-se que poucos são os trabalhos com amostras homogêneas e que isto, portanto, dificulta a generalização dos resultados encontrados, contribuindo para criar dúvidas sobre a eficácia da reabilitação em disfagia orofaríngea ${ }^{24-28}$,

Tendo em vista a escassez de trabalhos científicos sobre a eficácia da reabilitação em disfagia, sendo que somente recentemente importantes profissionais na atuação com disfagia orofaríngea uniram-se para emitir parecer sobre a eficácia da reabilitação ${ }^{29}$, este estudo teve por objetivo propor um protocolo para mensurar a eficácia da reabilitação fonoaudiológica na disfagia orofaríngea neurogênica.

\section{MÉTODOS}

O protocolo para controle de eficácia terapêutica em disfagia orofaríngea neurogênica (PROCEDON) foi proposto tendo em vista a concordância atual da literatura especializada sobre os parâmetros relevantes para mensurar a biomecânica da deglutição, o nível de ingestão oral e a percepção do indivíduo pré e pós-fonoterapia. Os parâmetros selecionados para compor o protocolo para controle de eficácia em disfagia orofaríngea foram divididos em dois blocos: a) trata da biomecânica da deglutição e utiliza o grau de comprometimento das disfagias e a medida do tempo de trânsito faríngeo por meio de análise quantitativa com software, b) engloba os benefícios conquistados para a ingestão oral segura de alimentos bem como a percepção do cliente.

Para ilustrar a aplicação do protocolo que está sendo proposto apresentaremos o controle de eficácia realizado com o referido protocolo em indivíduo Pós-AVE.

Participou deste estudo 1 indivíduo Pós-AVE isquêmico à direita, neuroimagem por tomografia computadorizada de crânio, 66 anos, gênero masculino, grau 1 incompleto, ausência de distúrbios cognitivos associados, presença de disartrofonia, apresentando disfagia orofaríngea grave, caracterizada por aspiração laringotraqueal silente para três consistências testadas (pastoso fino, líquido engrossado e líquido), em uso exclusivo de sonda nasoentérica pré-fonoterapia.

O programa de reabilitação foi desenvolvido durante 6 meses, conduzido por fonoaudiólogo com dois anos de formação específica em disfagia orofaríngea. Foram realizadas 15 sessões de 40 minutos com aplicação das técnicas pelo fonoaudiólogo, acompanhadas obrigatoriamente de um agente reabilitador. Foram realizadas sessões diárias pelo agente reabilitador, com controle sistemático gerenciado pelo fonoaudiólogo. Após análise funcional da deglutição, elaboramos a hipótese que dirigiu as condutas terapêuticas. O programa de reabilitação incluiu terapia indireta com exercícios orofaciais específicos de língua e terapia direta, com manipulação de consistências e volumes, utilizando técnicas de estimulação térmica fria, deglutição de esforço, deglutição múltipla e cabeça fletida.

O protocolo proposto para controle da eficácia terapêutica do programa de reabilitação fonoaudiológica foi aplicado, pré e pós-fonoterapia, contendo quatro distintos procedimentos: 1 - Classificação do grau de comprometimento da disfagia orofaríngea, 2 - Aplicação da Functional Oral Intake Scale ${ }^{13}$ (Figura 1), 3 - Avaliação videofluoroscópica da deglutição com a medida do tempo de trânsito faríngeo da deglutição por meio de software, 4 - Classificação da percepção dos familiares e pacientes sobre a deglutição.

1 - A partir dos achados clínicos o grau de comprometimento da disfagia orofaríngea do indivíduo foi classificada segundo os critérios propostos por Silva (1997) ${ }^{7}$. 
- Disfagia leve: alteração do esfíncter labial, incoordenação de língua, atraso para desencadear o reflexo de deglutição, ausência de tosse, sem redução acentuada da elevação da laringe, sem alteração da qualidade vocal após a deglutição e ausculta cervical sem alteração.

- Disfagia moderada: alteração do esfíncter labial, incoordenação de língua, atraso do reflexo de deglutição, ausência de tosse, presença de tosse antes, durante ou após a deglutição.

- Disfagia grave: atraso ou ausência do reflexo de deglutição, redução na elevação da laringe, ausência de tosse, presença de tosse antes, durante ou após a deglutição, alteração da qualidade vocal após a deglutição, alteração respiratória evidente, deglutição incompleta e ausculta cervical alterada.

2 - Para mensurar o grau de ingestão oral pré e pós-fonoterapia foi aplicada a Functional oral intake scale (FOIS) ${ }^{13}$ contendo sete níveis (Figura 1).

3 - A avaliação videofluoroscópica da deglutição com a medida do tempo de trânsito faríngeo da deglutição foi realizada mediante a metodologia proposta por Logemann (1983) ${ }^{30}$.

A partir dos achados videofluoroscópicos da deglutição o grau de comprometimento clínico da disfagia orofaríngea do indivíduo foi confirmado pela classificada segundo os critérios propostos por Ott et al (1996) ${ }^{9}$.

- Disfagia Leve: alteração do controle oral, atraso da resposta faríngea, pouco resíduo, sem penetração ou aspiração laringotraqueal.

- Disfagia Moderada: pobre controle oral, resíduo faríngeo em todas as consistências e pouca penetração ou aspiração laringotraqueal de uma consistência.

- Disfagia Grave: presença de aspiração laringotraqueal substancial ou quando ou paciente falha em deglutir.

As imagens digitalizadas foram submetidas à análise quantitativa por meio do software proposto por Spadotto et al (2008) ${ }^{15}$ e aplicado por Cola $(2008)^{31,32}$. Foi considerado tempo de trânsito farín- geo normal 1 segundo ou menos. Para análise computadorizada do tempo do trânsito faríngeo foi utilizado software que proporciona o registro do tempo em milissegundos, através da análise dos quadros do vídeo e da seriação da deglutição. Foi realizada análise quadro-a-quadro do exame, onde foi marcado o início e o término do trajeto do bolo pela fase faríngea da deglutição, obtendo-se assim o tempo de duração desta fase, através da contagem dos quadros. Considerou-se como início da fase faríngea da deglutição o momento em que o bolo alimentar atingia a região posterior da espinha nasal, localizada no final do palato duro, início do palato mole. Considerou-se como término da fase faríngea da deglutição o momento em que o bolo alimentar passava pelo esfíncter superior do esôfago ${ }^{14}$.

4 - A percepção da deglutição foi avaliada préfonoterapia e pós-fonoterapia, tendo o indivíduo que classificar sua deglutição conforme os critérios abaixo expostos. A entrevista após a fonoterapia foi realizada ao término da reabilitação e também 5 meses após a alta por meio de contato telefônico. A percepção do indivíduo foi classificada segundo os critérios abaixo expostos.

- Deglutição ruim: muita dificuldade para deglutir.

- Deglutição boa: dificuldade para deglutir determinados alimentos.

- Deglutição excelente: ausência de dificuldades para deglutir qualquer alimento.

O paciente permitiu a realização deste estudo, assim como a divulgação dos resultados, tendo este trabalho obtido aprovação do Comitê de Ética em Pesquisa da Instituição, sob número 10-2006.

Não foi utilizada análise estatística com testes paramétricos, sendo os resultados apresentados de forma descritiva.

\section{RESULTADOS}

Os resultados da mensuração pré-fonoterapia podem ser observados nas Figuras 2 e 3.

Os resultados da mensuração pós-fonoterapia podem ser observados nas Figuras 4 e 5.

( ) Nível 1: Nada por via oral

( ) Nível 2: Dependente de via alternativa com mínima via oral de alimento ou líquido

( ) Nível 3: Dependente de via alternativa com consistente via oral de alimento ou líquido

( ) Nível 4: Via oral total de uma única consistência

( ) Nível 5: Via oral total com múltiplas consistências, mas com necessidade de preparo especial ou compensações

( ) Nível 6: Via oral total com múltiplas consistências, mas sem necessidade de preparo especial ou compensações, porém com restrições para alguns alimentos

( ) Nível 7: Via oral total sem restrições.

${ }^{*}$ Functional oral intake scale (FOIS) 13 traduzido por Silva et al, 200623. 


\begin{tabular}{|l|c|}
\hline Classificação da Disfagia & Grave \\
\hline FOIS & 1 \\
\hline Tempo de trânsito faríngeo (TTF) & 13 segundos \\
\hline Percepção do cliente & Ruim \\
\hline
\end{tabular}

Figura 2 - Descrição dos parâmetros de mensuração aplicados pré-fonoterapia

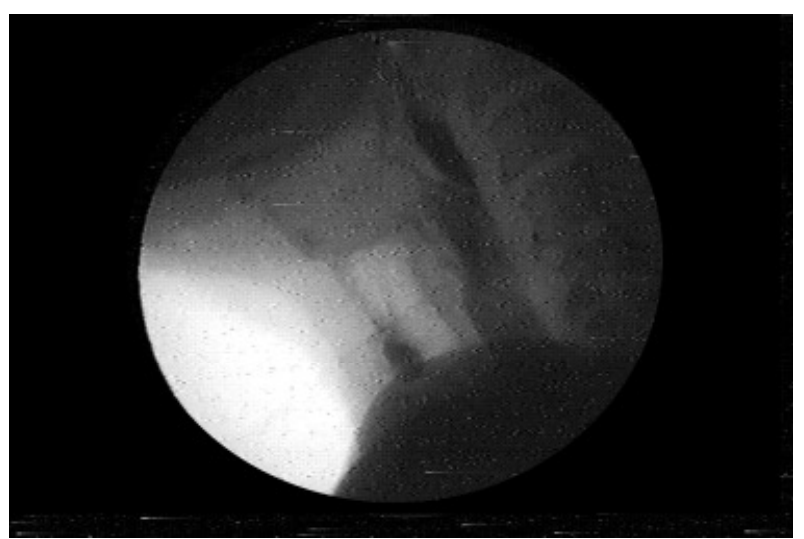

Fonte: Centro de Diagnóstico por Imagem do Hospital Estadual Bauru.

Figura 3 - Imagem da videofluoroscopia de deglutição pré-fonoterapia evidenciando aspiração laringotraqueal

\begin{tabular}{|l|c|}
\hline Classificação da Disfagia & Moderada \\
\hline FOIS & 5 \\
\hline Tempo de trânsito faríngeo (TTF) & 4 segundos \\
\hline Percepção do cliente & Boa \\
\hline
\end{tabular}

Figura 4 - Descrição dos parâmetros de mensuração aplicados pós-fonoterapia

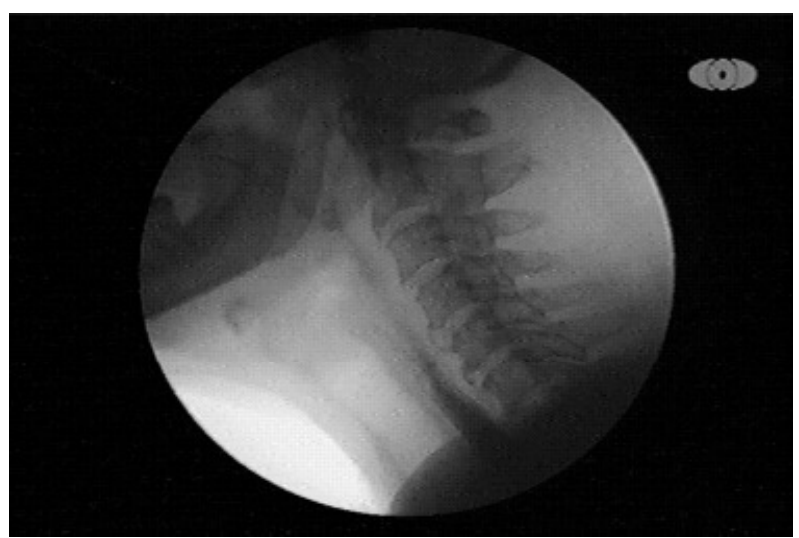

Fonte: Centro de Diagnóstico por Imagem do Hospital Estadual Bauru.

Figura 5 - Imagem da videofluoroscopia de deglutição pós-fonoterapia evidenciando ausência de aspiração laringotraqueal

\section{DISCUSSÃO}

Discussões recentes sobre a eficácia da reabilitação em disfagia orofaríngea retratam evidências da atuação fonoaudiológica, porém discutem a necessidade de novas pesquisas sobre esta temática ${ }^{33}$.

A proposição deste protocolo para mensurar a eficácia da reabilitação, agrupando a biomecânica da deglutição, o nível de ingestão oral e a percepção do cliente, reflete a concepção atual sobre a reabilitação em disfagia orofaríngea após minuciosa análise dos parâmetros apresentados pela literatura. Além disto, a conquista de um desses parâmetros isoladamente pode não causar impacto favorável para a qualidade de vida do paciente. A qualidade de vida do paciente disfágico tem sido uma preocupação mundial na atuação com disfagia orofaríngea ${ }^{34-36}$.

A mudança do grau de comprometimento no quadro disfágico deste paciente, caracterizada pela presença de disfagia grave pré-fonoterapia e moderada pós-fonoterapia, respectivamente mostradas nas Figuras 2 e 4, comprova as mudanças conquistadas na dinâmica da deglutição e a possibilidade das escalas de grau de comprometimento contribuírem para mensurar a evolução terapêutica. A maioria das classificações atualmente existentes para disfagia orofaríngea está baseada em critérios que privilegiam a presença ou não de penetração laríngea e aspiração laringotraqueal e, portanto, variações entre os distintos graus durante o processo terapêutico normalmente quer apenas sugerir ou confirmar o risco de aspiração ${ }^{6-12}$. Somente uma das classificações encontradas inclui critérios nutricionais ou pulmonares, sendo que não foram encontradas classificações de grau de comprometimento em disfagia que utilizem como critério o impacto para a qualidade de vida do paciente.

O conceito de leve, moderado e grave para a classificação do grau de comprometimento da disfagia orofaríngea na literatura atual foi baseado em critérios clínicos ou instrumentais referentes, exclusivamente, aos sinais sugestivos de penetração e aspiração laringotraqueal. Assim, atualmente há uma associação entre o grau de comprometimento da disfagia e a possibilidade de liberação de via oral que é norteada somente por critérios da fase faríngea da deglutição. No entanto, muitas disfagias classificadas como leve em pacientes pósAVE devido a ausência de aspiração podem possuir tempo de trânsito oral bastante aumentado, impossibilitando a via oral total por comprometer a condição nutricional.

Portanto, a classificação da disfagia não deve ser o único parâmetro a nortear a conduta frente à 
liberação de via oral parcial ou total, uma vez que as classificações atuais não pontuam nenhuma gravidade para os casos com tempo de trânsito oral (TTO) aumentado, e estes obterão prognóstico nutricional desfavorável mesmo podendo se alimentar por via oral. Além disto, nossa experiência já mostrou que é necessário ponderar de forma multifatorial sobre os casos que tem penetração laríngea e aspiram.

Outro parâmetro, ainda dentro das mudanças que podem ocorrer pré e pós-fonoterapia na dinâmica da deglutição, é a utilização da medida do tempo de trânsito faríngeo (TTF). Pré-fonterapia a deglutição do paciente apresentava 13 segundos de TTF, quando na fisiologia normal este tempo não excede de 1 a 2 segundos, caracterizando a grande dificuldade do paciente em desencadear a resposta faríngea. Em pacientes pós-AVE, a alteração de TTF já foi discutida, tanto com relação à mudança no tempo como também sobre os marcadores que definem o início da fase faríngea ${ }^{14,31,32}$. No entanto, não foram encontrados trabalhos na literatura que tivessem utilizado os softwares de medição de tempo de trânsito orofaríngeo para avaliar o desempenho da dinâmica da deglutição pré e pós-fonoterapia.

A fim de mensurar os resultados terapêuticos na disfagia orofaríngea associou-se aos resultados encontrados na dinâmica da deglutição, à aplicação da FOIS e da análise da percepção do paciente sobre sua deglutição pré e pós-fonoterapia.

Quanto a FOIS, o nível de ingestão oral mudou de 1 (nada por via oral), portanto uso exclusivo de via alternativa, para 5 (via oral total com múltiplas consistências, mas com necessidade de preparo especial ou compensações), comprovando a possibilidade de um paciente pós-AVE isquêmico à direita após a fase de recuperação espontânea, e mediante fonoterapia, alcançar via oral total gerenciada. No Brasil e em outros países, outros autores já utilizaram a FOIS como marcador para mensurar a eficácia da reabilitação em disfagia orofaríngea ${ }^{37-39}$. Entende-se, no entanto, que a avaliação nutricional deve vir associada à utilização da FOIS, para que seja possível um monitoramento eficiente das condições clínicas do paciente, agora em uso de via oral parcial ou total, sendo necessária a inclusão de parâmetros nutricionais nos protocolos de controle de eficácia na reabilitação da disfagia.

Todo processo de reabilitação é conduzido pelo profissional e por ele redirecionado, no entanto, o paciente necessita expor suas percepções e expectativas, para que os objetivos propostos possam impactar de forma benéfica a qualidade de vida do cliente. Além disto, a percepção do paciente pré e pós-fonoterapia é de fundamental importância para que o terapeuta eleja tanto os novos objetivos terapêuticos como os critérios de alta. Neste estudo, conforme mostram as Figuras 2 e 4, respectivamente, o paciente iniciou a reabilitação identificando a sua deglutição como ruim e finalizou o processo a classificando como boa.

Na comparação qualitativa da FOIS e da percepção do cliente, pode-se visualizar que a ausência de via oral foi percebida pelo paciente como ruim e a presença dela, mesmo que com restrições, eleva sua percepção para uma deglutição boa. Nada mais natural do que o ser humano perceber como boa a possibilidade de alimentar-se pela boca.

Por fim, a possibilidade de mensurar os resultados da fonoterapia em disfagia orofaríngea é uma área bastante promissora, necessitando ainda de inúmeras pesquisas, independente dos critérios ou protocolos utilizados para este fim, bastando, no entanto, que o terapeuta tenha mais informações a fornecer após o processo de reabilitação do que somente observações subjetivas.

\section{CONCLUSÃO}

O protocolo proposto foi capaz de avaliar a eficácia da reabilitação na disfagia orofaríngea neurogênica neste indivíduo pós-acidente vascular encefálico, tanto para mensurar as mudanças ocorridas na fisiopatologia da deglutição quanto na ingestão oral e na percepção do indivíduo. Ainda são necessárias inúmeras pesquisas com amostras controladas, sugerindo-se ainda a inclusão da condição nutricional e pulmonar do indivíduo no controle de eficácia em disfagia orofaríngea. 


\section{ABSTRACT}

Purpose: to submit a proposal for efficacy control in neurogenic oropharyngeal dysphagia. Methods: the protocol was proposed in accordance with other researches and applied in one post-stroke individual, with right-hemispheric lesion confirmed by computed tomography, with oropharyngeal dysphagia, male gender, 66-year old, with laringotraqueal aspiration and using nasogastric feeding tube before swallowing therapy. In order to control the therapy effectiveness in pre- and post-swallowing therapy, the following procedures were applied: classification of severity degree for oropharyngeal dysphagia, functional oral intake scale (FOIS), videofluoroscopic evaluation of swallowing with additional swallowing pharyngeal transit time evaluation using a specific software and individual perception. Results: before swallowing therapy, severe oropharyngeal dysphagia, FOIS level 1, presence of laryngotracheal aspiration, and 13 seconds of pharyngeal transit time were found. After swallowing therapy, moderate oropharyngeal dysphagia, FOIS level 5, absence of laryngotracheal aspiration, and 4 seconds of pharyngeal transit time were found. Conclusion: the proposed protocol could measure changes, both for pathophysiology of swallowing as well as for oral ingestion of the individual. We believe that it is still necessary to include nutritional and lung status of the individual in efficacy control of oropharyngeal dysphagia.

KEYWORDS: Deglutition Disorders; Rehabilitation; Efficacy

\section{REFERÊNCIAS}

1. Morgan AT, Ward EC, Murdoch BE. A case study of the resolution of paediatric dysphagia following brainstem injury: clinical and instrumental assessment. J Clin Neurosci. 2004; 11(2):182-90.

2. Bedgood R, Bullock M, Chaudhary A. A case of occult oropharyngeal dysphagia. (Letters to the Editor). South Med J. 2006; 99(5):548.

3. Langmore SE. Issues in the management of dysphagia. Folia Phoniatr Logop. 1999; 51(4-5):220-30.

4. Skeat J, Perry A. Outcome measurement in dysphagia: not so hard to swallow. Dysphagia. 2005; 20(2):113-22.

5. Silva RG. A eficácia da reabilitação em disfagia orofaríngea. Pró-Fono. 2007; 19(1):123-30.

6. Daniels SK, McAdam CP, Brailey K, Foundas AL. Clinical assessment of swallowing and prediction of dysphagia severity. Am J Speech Lang Pathol. 1997; 6(4):17-2.

7. Silva RG. Disfagia neurogênica em adultos pós - acidente vascular encefálico: identificação e classificação. [dissertação]. São Paulo (SP): Universidade Federal de São Paulo; 1997.

8. Silva RG. Disfagia orofaríngea pós-acidente vascular encefálico. In: Ferreira LP, Befi-Lopes DM, Limongi SCO, organizadores. Tratado de fonoaudiologia. São Paulo: Rocca; 2004. p. 354-69. 9. Ott DJ, Hodge RG, Pikna LA, Chen MY, Gelfand DW. Modified barium swallow: clinical and radiographic correlation and relation to feeding recommendations. Dysphagia. 1996; 11(3):187-90.
10. Salassa JR. A functional outcome swallowing scale for staging oropharyngeal dysphagia. Dig Dis. 1999; 17(4):230-4.

11. O'Neil KH, Purdy M, Falk J, Gallo L. The dysphagia outcome and severity scale. Dysphagia. 1999; 14(3):139-45.

12. Padovani AR, Moraes DP, Mangili LD, Andrade CRF. Protocolo fonoaudiológico de avaliação do risco para disfagia (PARD). Rev Soc Bras Fonoaudiol. 2007; 12(3):199-205.

13. Crary MA, Mann GD, Groher ME. Initial psychometric assessment of a functional oral intake scale for dysphagia in stroke patients. Arch Phys Med Rehabil. 2005; 86(8):1516-20.

14. Kendall KA, McKenzie S, Leonard RJ, Gonçalves MI, Walker A. Timing of events in normal swallowing: a videofluoroscopic study. Dysphagia. 2000; 15(2):74-83.

15. Spadotto AA, Gatto AR, Cola PC, Montagnoli $A N$, Schelp AO, Silva RG, et al. Software para análise quantitativa da deglutição. Radiol Bras. 2008; 41(1):25-8.

16. Silva RG, Jorge AG, Peres FM, Cola PC, Gatto AR, Spadotto AA. Efficacy protocol in a oropharyngeal dysphagia: a proposal. XVII Dysphagia Research Society Meeting; 2009, Março 5-7; New Orleans-Lousiana, EUA.

17. Schelp AO, Cola PC, Gatto AR, Silva RG, Carvalho LR. Incidência de disfagia orofaríngea após acidente vascular encefálico em hospital público de referência. Arq Neuropsiquiatr. 2004; 62(2b):503-6. 
18. Broadley S, Cheek A, Salonikis S, Whitham E, Chong V, Cardone D, et al. Predicting prolonged dysphagia in acute stroke: the Royal Adelaide Prognostic Index for Dysphagic Stroke (RAPIDS). Dysphagia. 2005; 20(4):303-10.

19. Martino R, Foley N, Bhogal S, Diamant N, Speechley M, Teasell R. Dysphagia after stroke: incidence, diagnosis, and pulmonary complications. Stroke. 2005; 36(12):2756-63.

20. Singh S, Hamdy S. Dysphagia in stroke patients. Postgraduate Med J. 2006; 82(968):383-91.

21. Kasprisin AT, Clumeck $H$, Nino-Murcia $M$. Efficacy of rehabilitative management of dysphagia. Dysphagia. 1989; 4(1):48-52.

22. Bath PMW, Bath-Hextall FJ, Smithard D. Interventions for dysphagia in acute stroke. Cochrane Database Syst Rev. 2000; (2):CD000323. DOI: $10.1002 / 14651858$.

23. Silva RG, Jorge AG, Peres MA. A eficácia da reabilitação em disfagia orofaríngea neurogênica: estudo de caso. XIV Congresso Brasileiro de Fonoaudiologia; 2006, Outubro 4-7; Salvador, BA. p.294.

24. Bartolome G, Neumann S. Swallowing therapy in patients with neurological disorders causing cricopharyngeal dysfunction. Dysphagia. 1993; 8(2):146-9.

25. Miller RM Langmore SE. Treatment efficacy for adults with oropharyngeal dysphagia. Arch Phys Med Rehabil. 1994; 75(11):1256-62.

26. Hill M, Hughes $T$, Milford C. Treatment for swallowing difficulties (dysphagia) in chronic muscle disease. Cochrane Database Syst Rev. 2004; (2):CD004303. DOI: 10.1002/14651858.

27. Nguyen NP, Moltz CC, Frank C, Karlsson U, Smith HJ, Nguyen PD, et al. Severity and duration of chronic dysphagia following treatment for head and neck cancer. Anticancer Res. 2005; 25(4):2929-34.

RECEBIDO EM: 15/04/2009

ACEITO EM: 01/10/2009

Endereço para correspondência:

Roberta Gonçalves da Silva

Universidade Estadual Paulista-UNESP

Departamento de Fonoaudiologia

Av. Hygino Muzzi Filho, 737

Marília - SP

CEP: $17525-900$

E-mail: rgsilva@marilia.unesp.br
28. Prosiegel $M$, Höling $R$, Heintze $M$, WagnerSonntag E, Wiseman K. Swallowing therapy: a prospective study on patients with neurogenic dysphagia due to unilateral paresis of the vagal nerve, Avellis' syndrome, Wallenberg's syndrome, posterior fossa tumours and cerebellar hemorrhage. Acta Neurochir Suppl. 2005; 93:35-7.

29. Coyle J, Davis L, Easterling C, Graner D, Langmore S, Leder $S$, et al. Oropharyngeal dysphagia assessment and treatment efficacy: setting the record straight. J Am Med Dir Assoc. 2009; 10(1):62-6.

30. Logemann JA. Evaluation and treatment of swallowing disorders. San Diego: College Hill Press; 1983.

31. Cola PC. O efeito do sabor azedo e da temperatura fria no tempo de trânsito faríngeo da deglutição em indivíduos após acidente vascular encefálico hemisférico [dissertação]. Botucatu (SP): Universidade Estadual Paulista; 2007.

32. Cola PC, Gatto AR, Silva RG, Spadotto AA, Schelp AO, Henry MAA. The influence of sour taste and cold temperature on pharyngeal transit time in cerebral vascular accident patients. Arq Gastroenterol. 2009. No prelo.

33. Logemann JA. Treatment of oral and pharyngeal dysphagia. Phys Med Rehabil Clin N Am. 2008; 19(4):803-16.

34. Costa BAK, Azevedo EH, Vartanian JG, Nishimoto IN, Kowaslki LP, Carrara-de Angelis E. Quality of life related to swallowing after tongue cancer treatment. Dysphagia. 2008; 23(2):189-92.

35. Khaldoun E, Woisard V, Verin E. Validation in French of the SWAL-QOL scale in patients with oropharyngeal dysphagia. Gastroenterol Clin Biol. 2009; 33(3):167-71.

36. Bogaardt HC, Speyer R, Baijens LW, Fokkens WJ. Cross-cultural adaptation and validation of the Dutch version of SWAL-QoL. Dysphagia. 2009; 24(1):66-70.

37. Hansen TS, Larsen K, Engberg AW. The association of functional oral intake and pneumonia in patients with severe traumatic brain injury. Arch Phys Med Rehabil. 2008; 89(11):2114-20.

38. Furkim AM, Sacco ABF. Eficácia da fonoterapia em disfagia neurogênica usando a escala funcional de ingestão por via oral (FOIS) como marcador. Rev CEFAC. 2008; 10(4):503-12. dx.doi.org/10.1590/ S1516-18462008000400010

39. Crary MA, Carnaby GD, Groher ME, Helseth E. Functional benefits of dysphagia therapy using adjunctive sEMG biofeedback. Dysphagia. 2004; 19(3):160-4. 